\title{
Fast simulation of phase-change processes in chalcogenide alloys using a Gillespie-type cellular automata approach
}

\author{
Peter Ashwin, ${ }^{1}$ B. S. V. Patnaik, ${ }^{2, a)}$ and C. David Wright ${ }^{1}$ \\ ${ }^{1}$ School of Engineering, Computing and Mathematics, University of Exeter, Harrison Building, Exeter EX4 \\ $4 Q F$, United Kingdom \\ ${ }^{2}$ Department of Applied Mechanics, Indian Institute of Technology, Madras, Chennai 600 036, India
}

(Received 12 May 2008; accepted 16 July 2008; published online 20 October 2008)

\begin{abstract}
A stochastic cellular automata simulator capable of spatiotemporal modeling of the crystallization and amorphization behavior of phase-change materials during the complex annealing cycles used in optical and electrical memory applications is presented. This is based on consideration of bulk and surface energies to generate rates of growth and decay of crystallites built up from "monomers" that may themselves be quite complex molecules. The approach uses a stochastic Gillespie-type time-stepping algorithm to deal with events that may occur on a very wide range of time scales. The simulations are performed at molecular length scale and using an approximation of local free energy changes that depend only on immediate neighbors. The approach is potentially capable of spanning the length scales between ab initio atomistic modeling methods, such as density functional theory, and bulk-scale methods, such the Johnshon-Mehl-Avrami-Kolmogorov formalism. As an example the model is used to predict the crystallization behavior in the chalcogenide $\mathrm{Ge}_{2} \mathrm{Sb}_{2} \mathrm{Te}_{5}$ alloy commonly used in phase-change memory devices. The simulations include annealing cycles with nontrivial spatial and temporal variations in temperature, with good agreement to experimental incubation times at low temperatures while modeling nontrivial crystal size distributions and melting dynamics at higher temperatures. (C) 2008 American Institute of Physics.
\end{abstract}

[DOI: 10.1063/1.2978334]

\section{INTRODUCTION}

Over the past 20 years and more there has been great interest in chalcogenide materials, such as GeSbTe (GST) or AgInSbTe alloys, for a range of data storage applications. Currently the most prominent and widespread use of such materials is in the rewritable phase-change optical memory disks, such as CD-RW, DVD+/-RW, etc. Their use in nonvolatile, solid-state, electrical memories, first investigated nearly 40 years ago, is also of much interest, ${ }^{1}$ as they offer a possible replacement for the conventional silicon-based flash memory currently used in USB memory sticks and other memory card formats. The use of phase-change materials for scanning-probe-based storage is also possible. ${ }^{2}$ All of these memory applications rely on a reversible phase transformation of the chalcogenide alloy between the amorphous and (poly)crystalline states. In optical disk memories such phase transformations are brought about by heating with a focused laser; readout relies on the different optical reflectivities of the two phases. In electrical phase-change memories, data are written or erased by resistive heating caused by a pulse of electrical current injected into the phase-change layer; readout relying on sensing the different electrical resistivities of the two phases (that can differ by more than a factor of a thousand). Thus, it is clear that phase-change materials have many technologically important applications, and it is therefore necessary and desirable to have a thorough understanding of the phase-transformation processes that make these materials so useful.

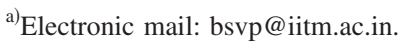

Several methods have been used over the years to model the process of crystallization in phase-change alloys, such as the Johnson-Mehl-Avrami-Kolmogorov (JMAK) model. Unfortunately many of the assumptions on which JMAK formalism is based are violated in real switching events in phase-change devices. ${ }^{3}$ Furthermore JMAK cannot distinguish materials with the same crystallized fraction but different crystallite size distributions, and this is important to predict the dynamical progress of the complex annealings often necessary for memory applications. Another common modeling approach is based on separable nucleation and growth models, often used to examine optical phase-change recording. ${ }^{4}$ However, such approaches deal only with crystal clusters at or above the critical (stable) size, whereas subcritical clusters are also likely to play a significant role in the nanoscale dynamic behavior of future devices. An attractive, physically plausible, alternative to these more established methods is the rate-equation approach that models the evolution of the crystal cluster size distribution during the entire phase-transformation process. Using rate equations, the frequencies of attachment and detachment of material "monomers" representing unit changes in crystal cluster sizes can be determined. We have investigated the use of both discrete and continuous versions of the rate-equation approach to model phase transformations in $\mathrm{Ge}_{2} \mathrm{Sb}_{2} \mathrm{Te}_{5}$, and they have proved extremely useful in predicting bulk annealing behaviour. ${ }^{3,5}$ However, the rate-equation approach is computationally intensive and not directly suited to cases, such as optical and electrical memories, where understanding the spatial distribution of crystallization is important. 
More recently, $a b$ initio atomic-scale models of crystallization processes in phase-change materials have been developed based on the well-known density function theory (DFT) approach. ${ }^{6,7}$ However, DFT-type approaches come at a steep computational price, and only arrangements of a few tens of atoms can be simulated without supercomputing resources, making such approaches unsuited to understanding and predicting the details of real device operation and for use in real device design.

This paper presents a modeling approach that is potentially capable of spanning the scales between atomistic methods, such as DFT, and bulk-scale methods, such the JMAK formalism. The model combines the attractive (thermodynamic) features of the rate-equation approach with elements from probabilistic cellular automata models, ${ }^{8,9}$ and phasefield models. ${ }^{10-12}$ In addition, it uses the Gillespie algorithm for time stepping, so providing for fast simulation of complex spatial and temporal annealing (heating/cooling) cycles essential to the operation of current and future phase-change memory devices. A high level of simplicity is maintained by using a discrete time and lattice space model, while retaining thermodynamic realism and hence keeping fitting parameters to a minimum.

A two dimensional (2D) version of the model is presented here, with extension to three dimensional (3D) in principle, being computationally trivial. The material space to be modeled is thus described as a $2 \mathrm{D}$ lattice of discrete grid points, where each site is either crystalline or amorphous and there is an underlying orientation that varies continuously; these sites are on the length scale of monomers, although they do not necessarily correspond directly to individual monomers. Recall that crystallization can be thought of as a two-stage process; nucleation (where a small crystallite needs to overcome an energy barrier dominated by interfacial energy) and growth (where the crystallite grows according to the availability of neighboring monomers and dominated by bulk energy). Each site is assumed to have a set of locally determined rate constants for transitions into a new state. These rates depend only on the current state of the site and that of its immediate neighbors. For the rates of growth and dissociation for modeling of GST materials, thermodynamic parameters from previous work are used. ${ }^{3}$

The Gillespie algorithm ${ }^{13}$ can be used to simulate the evolution under the assumption that the events are independent, instantaneous, and never simultaneous. Each step of the algorithm has two parts; firstly it determines a random time increment to the next event and secondly it determines which event should occur. This enables fast and physically realistic simulations to be performed of a number of crystallizationrelated phenomena, including incomplete crystallization, melting, and complex spatiotemporal annealings. As there are many possible events, data structures must be used efficiently to ensure that the simulations run at a high speed and hence perform simulation of complex annealings in $2 \mathrm{D}$ on a standard desktop computer.

Section II describes the detail of our "Gillespie-type cellular automata" (GCA) simulation approach, while Sec. III presents the results of some annealings of GST using this simulator, showing that the simulator can model nucleation effects, nontrivial annealings, and melting. Examples are included where the temperature depends on space and/or time; one can see a variety of effects and a good quantitative agreement with experimental temperature-dependent incubation times for GST. Finally Sec. IV discusses some possible extensions and limitations of the method.

\section{THE GCA SIMULATOR}

A homogeneous, isotropic, material in two dimensions is considered on a square lattice. The state of the material is described on a discrete regular lattice of grid points $G$. Each lattice site is assumed to be either crystalline or amorphous. More precisely, at each grid point $(i, j) \in G$, the state is described by two quantities.

(1) $r_{i j}$ refers to a discrete "phase" variable that is either amorphous (or) crystalline. This is indicated by an integer (0) for amorphous and (1) for crystalline.

(2) $\phi_{i j}$ a continuous "orientation" variable that varies over some range of $0-\pi$, which in turn gives a notional representation of local orientation to the material. Such a modeling approach mimics the infinitely many possible orientations, reflecting a number of phase-field variables.

Two adjacent crystalline sites $(i, j)$ and $(k, l)$ are determined to be within the same crystal, when

$$
r_{i j}=r_{k l}=1 \text { and } \phi_{i j}=\phi_{k l}
$$

are satisfied. A stochastic model for the temporal evolutions is developed, by providing an estimate of rates of possible local changes to the state of the system (i.e., changes that affect only one site) by employing a Gillespie algorithm. ${ }^{13} \mathrm{~A}$ Gillespie algorithm is optimal in that it will generate time steps at a rate corresponding to the fastest rate that requires updating, although it is typically more complex to implement than a Monte Carlo simulation. ${ }^{14}$

The following possible instantaneous events at a site $(i, j) \in G$ are possible.

Nucleation. The site $(i, j)$ and an adjacent site, originally both amorphous, become a single crystal at a rate $C_{i j}^{\mathrm{nu}}$.

Growth. The site $(i, j)$, originally amorphous, becomes attached to an adjacent crystal of orientation $\psi$ at a rate $C_{i j \psi}^{\mathrm{gr}}$.

Dissociation. The site $(i, j)$, originally crystalline, detaches or dissociates from the crystal of which it is a part and separates to become amorphous at a rate $C_{i j}^{\mathrm{di}}$ and assumes a random orientation.

\section{A. The rate coefficients}

The rate coefficients for nucleation, growth, and dissociation given by $C^{\mathrm{nu}}, C^{\mathrm{gr}}$, and $C^{\mathrm{di}}$ are approximated at each grid point in the domain by consideration of the change to bulk and surface energies of crystallites adjacent to that site.

The set of neighbors of $(i, j) \in G$ are defined as $N_{i j}$ $=\{(k, l) \in G:(k, l)$ is a neighbor of $(i, j)\}$, and $n_{i j}=\left|N_{i j}^{\mathrm{am}}\right|$ the set of amorphous neighbors of $(i, j)$, 


$$
N_{i j}^{\mathrm{am}}=\left\{(k, l) \in N_{i j}: r_{k l}=0\right\} \quad \text { and } n_{i j}^{\mathrm{am}}=\left|N_{i j}^{\mathrm{am}}\right|,
$$

and finally the set of neighbors of $(i, j)$ with a given orientation $\psi$ are

$$
\begin{aligned}
N_{i j \psi}^{\mathrm{or}}= & \left\{(k, l) \in N_{i j}: \phi_{k l}=\psi \text { and } r_{k l}=1\right\}, \\
& \text { and } n_{i j \psi}^{\text {or }}=\left|N_{i j \psi l}^{\mathrm{or}}\right|,
\end{aligned}
$$

It should be noticed that $N_{i j}^{\mathrm{am}}, N_{i j \psi}^{\mathrm{or}} \in\left\{0, \ldots, n_{i j}\right\}$.

The rates are considered in a similar way to the derivation of master equation rates as in Ref. 3. The "interactions" (which model molecular collisions) are assumed to occur at a temperature-dependent rate,

$$
R(T)=k_{0} e^{\left(-E_{a} / k_{B} T\right)},
$$

where $E_{a}$ is an activation energy and $k_{B}$ is the Boltzmann constant. The prefactor $k_{0}$ is used as a fitting parameter to normalize the results. The detailed model is available in Ref. 3.

If adjacent sites have an interaction then $\xi(T, A)$ is the rate at which a site transforms from amorphous to crystalline, resulting in a change $\mathrm{A}$ in the surface area of a crystallite and we assume local thermal equilibrium, meaning that the rate of the reverse transformation at an interaction is $\xi^{-1}(T, A)$. This rate varies with temperature in a similar way to the master equation model ${ }^{3}$ as the bulk and surface energy vary. The change in surface area of the crystallites is computed by adding site $(i, j)$ to a neighboring crystal of orientation $\psi$ by a linear approximation,

$$
A=S_{m}\left[\frac{n_{i j}-2 n_{i j \psi}^{\text {or }}}{n_{i j}}\right],
$$

where $S_{m}$ is the surface area of a single site. This means that changing an isolated site in the middle of a crystal of orientation $\psi$ will result in a change $A=-S_{m}$ as $n_{i j}=n_{i j \psi}^{o r}$, while creating a new crystal in the middle of a field of amorphous material will result in a change $A=S_{m}$ as $n_{i j \psi}^{o r}=0$.

Putting this together and noting that only by interaction with amorphous neighbors, it is possible to get a site to nucleate, the rate coefficients for nucleation are therefore

$$
C_{i j}^{\mathrm{nu}}= \begin{cases}k_{0} e^{\left(-E_{a} / k_{B} T\right)} \frac{n_{i j}^{\mathrm{am}}}{n_{i j}} \xi\left(T, S_{m}\right), & \text { if } r_{i j}=0 \\ 0, & \text { if } r_{i j}=1\end{cases}
$$

The growth rate for an amorphous site to join a crystalline neighbor with orientation $\psi$ is

$$
C_{i j \psi}^{\mathrm{gr}}= \begin{cases}k_{0} e^{\left(-E_{a} / k_{B} T\right)} \xi\left(T, S_{m} \frac{n_{i j}-2 n_{i j \psi}^{\mathrm{or}}}{n_{i j}}\right), & \text { if } r_{i j}=0 \\ 0, & \text { if } r_{i j}=1 .\end{cases}
$$

Finally, the dissociation rate for a crystalline site to become amorphous is

$$
C_{i j \psi}^{\mathrm{di}}= \begin{cases}0, & \text { if } r_{i j}=0 \\ k_{0} e^{\left(-E_{a} / k_{B} T\right)} \xi\left(T, S_{m} \frac{n_{i j}-2 n_{i j \psi}^{\mathrm{or}}}{n_{i j}}\right)^{-1}, & \text { if } r_{i j}=1 .\end{cases}
$$

\section{B. The GCA algorithm}

The GCA algorithm that employs the rate coefficients specified above is as follows. Initially, the whole domain is assumed to be an as deposited amorphous state with a random distribution of $\phi_{i j}$ values and $r_{i j}=0$. The program is implemented in such a way that it is also possible to restart the algorithm from any given state.

Here, a square lattice is used and so eight neighbors are chosen for each grid point. But, these are weighted according to the inverse distance from the site. Note that the four corner sites are at a distance of $\sqrt{2}$ as they are along the diagonal. The new state of the site is then given by $r_{i j}^{\prime}$ and $\phi_{i j}^{\prime}$, using the stochastic simulation algorithm of Gillespie ${ }^{13}$ as follows. This simulates up to a time $T_{\max }$.

\section{Algorithmic steps}

(1) Start at time $T=0$ with given $r_{i j}$ and $\phi_{i j}$.

(2) Generate rate coefficients for all grid points $C_{i j}^{\mathrm{nu}}, C_{i j \psi}^{\mathrm{nu}}$, and $C_{i j}^{\mathrm{di}}$ for nucleation, growth, and dissociation, respectively. We refer to these using a single index $\nu=(i, j, a)$, where $a$ refers to an activity (or) an event $a$ $\in\{\mathrm{nu},(\mathrm{gr}, \psi), \mathrm{di}\}$.

(3) Compute the sum,

$a_{0}=\sum C_{i j}^{\mathrm{nu}}+\sum_{i j}\left[\sum_{\psi \in \Psi_{i j}} C_{i j \psi}^{\mathrm{gr}}\right]+C_{i j}^{\mathrm{di}}$,

where $\Psi_{i j}=\left\{\phi_{k l}:(k, l) \in N_{i j}\right\}$ is the set of orientations of neighbors to $(i, j)$.

(4) Generate two independent random numbers $\eta_{1}, \eta_{2}$ uniformly distributed on $(0,1)$ and compute $d \tau$ $=\left(1 / a_{0}\right) \log _{e}\left(1 / \eta_{1}\right)$. Increment time to $T=T+d \tau$. If $T$ $\geq T_{\max }$ then, stop.

(5) Identify the event $\nu=(i, j, a)$ corresponding to grid point $(i, j)$ and reaction (or) activity to be performed $a$ $\in\{$ nu, gr, di $\}$ and the $(k, l)$ with $\phi_{k l}=\psi$ based on the following:

$$
\sum_{\nu=1}^{\mu-1} a_{\nu}<\eta_{2} a_{0} \leq \sum_{\nu=1}^{\mu} a_{\nu} .
$$

(6) Update the value of $\phi_{i j}$ and $r_{i j}$. More precisely, perform

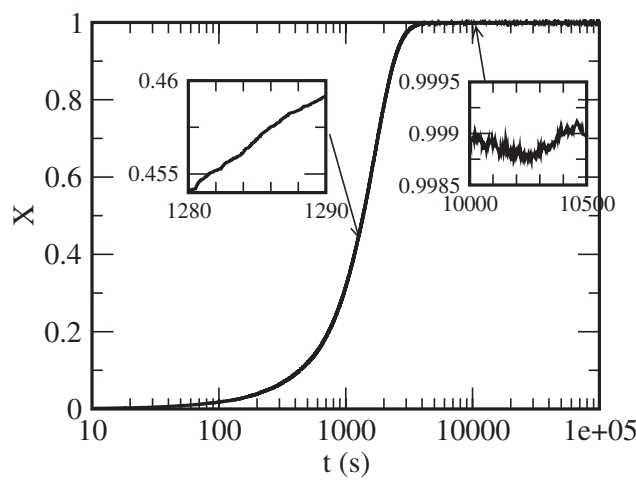

FIG. 1. Crystalline fraction $X$ as a function of time during low temperature annealings at $131^{\circ} \mathrm{C}$. Detail of the progress of the annealing is shown during the growth phase and when the crystalline fraction has saturated near $X=1$. 


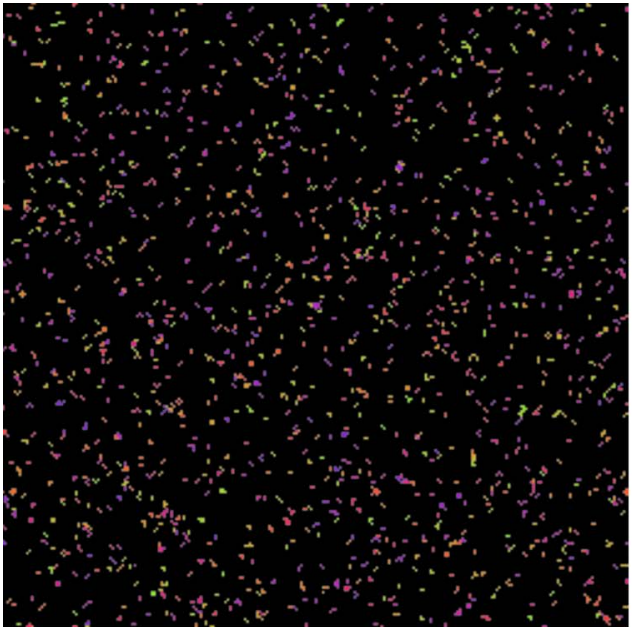

(a)

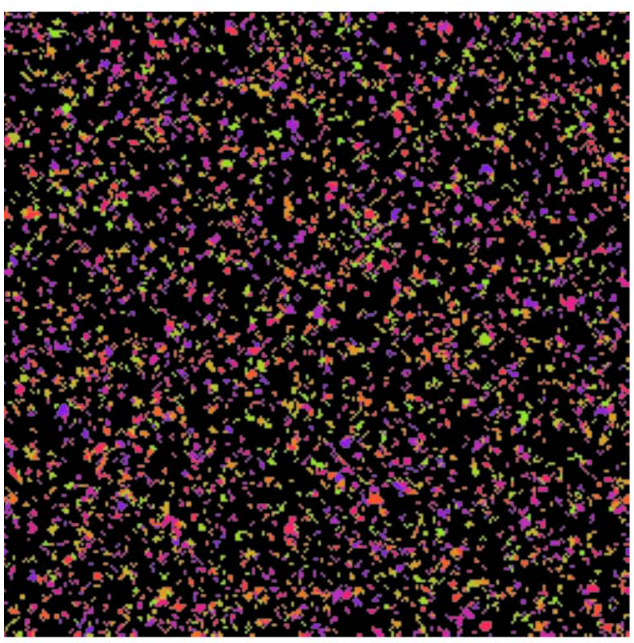

(b)

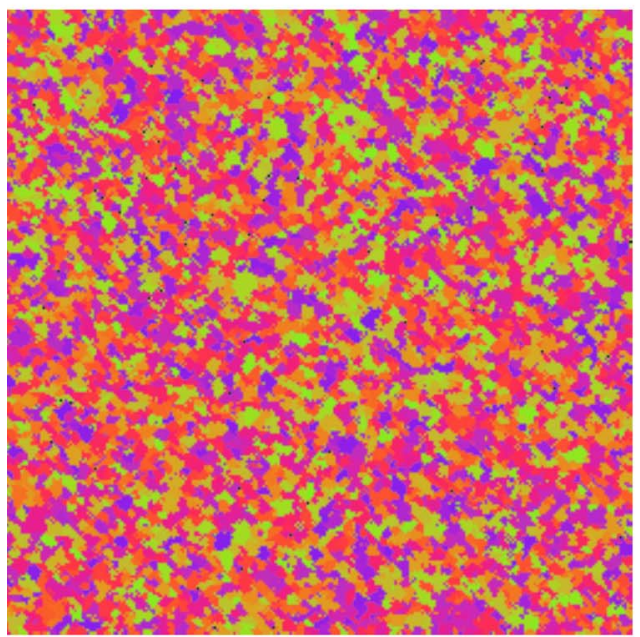

(c)

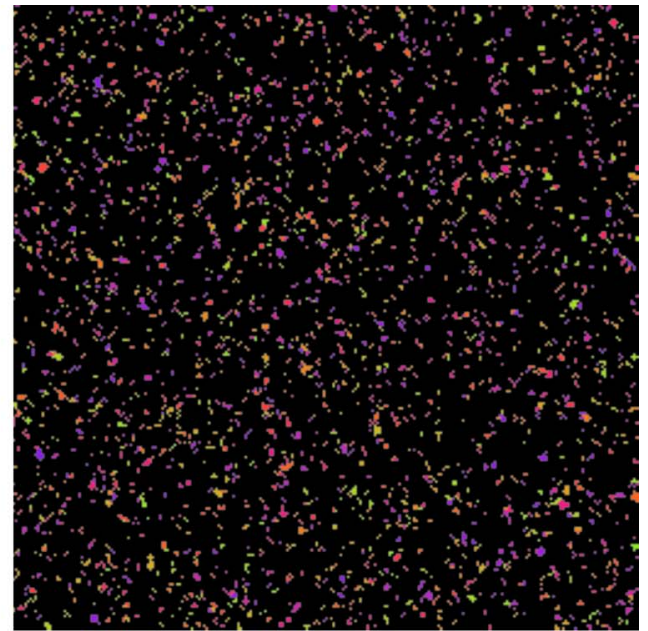

(d)

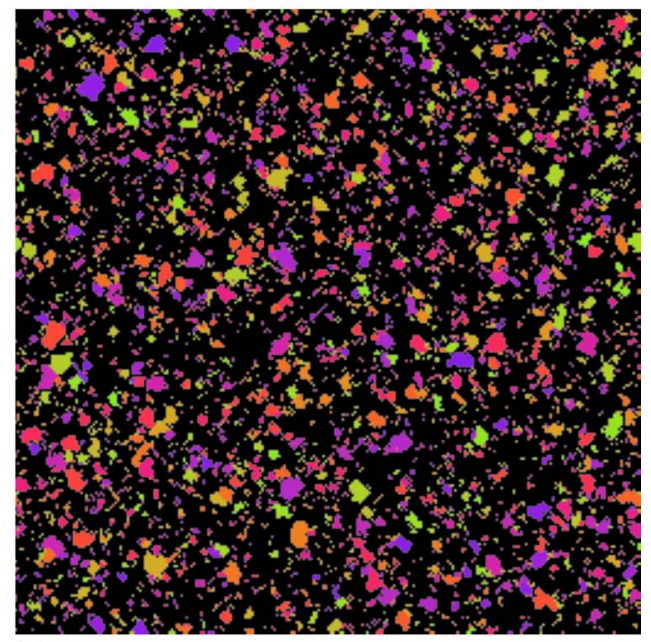

(e)

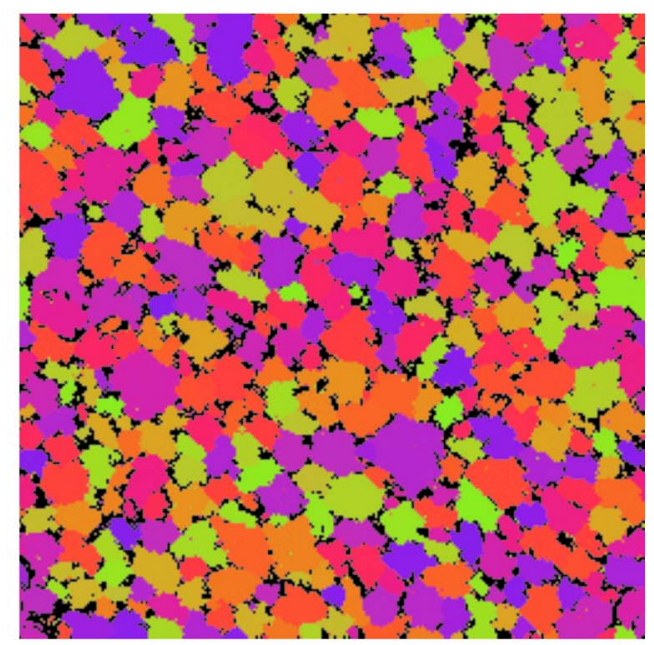

(f)

FIG. 2. (Color online) Images showing progress in crystallization $[(\mathrm{a})-(\mathrm{c})]$ for $T=131{ }^{\circ} \mathrm{C}$ (as shown in Fig. 1$)$ and $[(\mathrm{d})-(\mathrm{f})]$ for $T=407{ }^{\circ} \mathrm{C}$, starting with pure amorphous material. The colors are assigned arbitrarily to different oriented crystal grains. (a) shows after 2000 steps of the algorithm a number of nuclei with $X=0.0507$ after time of $248 \mathrm{~s}$, (b) shows after 10000 steps with $X=0.204$ after time of $743 \mathrm{~s}$, (c) shows after $10^{5}$ steps with $X=0.999115$ after time of 68930 s. Similarly, (d)-(f) show the state after $10^{4}, 10^{5}$, and $10^{6}$ steps corresponding to times of $0.145,0.679$, and $4.347 \mu$ s, respectively. Observe the faster progress and larger nucleation size for the higher temperature. 


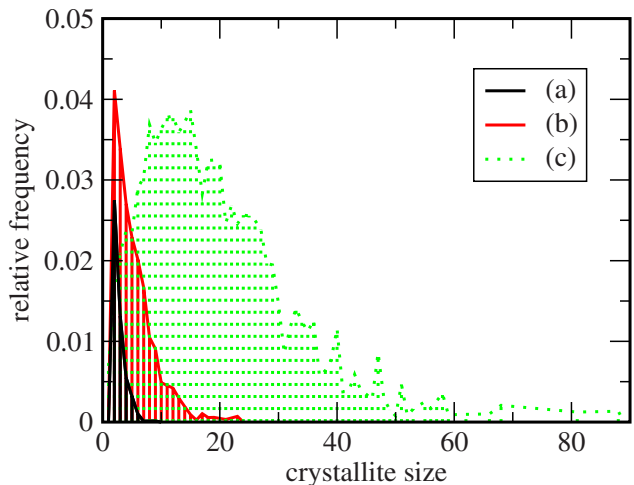

FIG. 3. (Color online) The relative frequency of crystallites of different sizes corresponding to the (a)-(c) of Fig. 2. Observe the peak in crystal size distribution at size of 10-15 $\mathrm{nm}$ for the fully developed crystal structure.

the following updates according to corresponding reactions (nucleation, growth, or dissociation) that occur.

(a) Nucleation at $(i, j)$ pick a $(k, l) \in N_{i j}^{\mathrm{am}}$ at random and set $\phi_{i j}^{\prime}=\phi_{k l}^{\prime}=\phi_{i j}, \quad r_{i j}^{\prime}=r_{k l}^{\prime}=1$.

(b) Growth from neighboring crystal with $\psi=\phi_{k l},(k, l)$ $\in N_{i j}$ into the amorphous site $(i, j)$; set $\phi_{i j}^{\prime}=\phi_{k l}, r_{i j}^{\prime}=1$.

(c) Dissociation at $(i, j)$, where we set $r_{i j}^{\prime}=0 \quad \phi_{i j}^{\prime}=W$, where $W$ is an independent random number uniformly distributed in the range of orientations $[0, \pi]$.

(7) For the next iteration, copy $\phi_{i j}=\phi_{i j}^{\prime}, r_{i j}=r_{i j}^{\prime}$ and update the values of $C_{i j}^{\mathrm{nu}}, C_{i j \psi}^{\mathrm{nu}}$ and $C_{i j}^{\mathrm{di}}$.

(8) Return to step 3 and recompute $a_{0}$.

Note that the main computational effort is actually in the selection of the event (step 5) based on $\eta_{2}$. However, to minimize the number of operations needed to determine this step, a recursive bisection search coupled with an efficient sorting scheme is implemented for all events. Also in the recomputation of rates (step 7) one can limit the updates to those sites that have changed and their neighbors. Finally, the computation of $a_{0}$ (step 3 ) in subsequent steps can be considerably accelerated by using only addition and subtraction of those rates that have changed.

\section{SIMULATION OF PHASE-CHANGE PROCESSES IN $\mathrm{Ge}_{2} \mathrm{Sb}_{2} \mathrm{Te}_{5}$}

For the remainder of this paper, the phase-change material GST is used to model the read/write optical and electrical

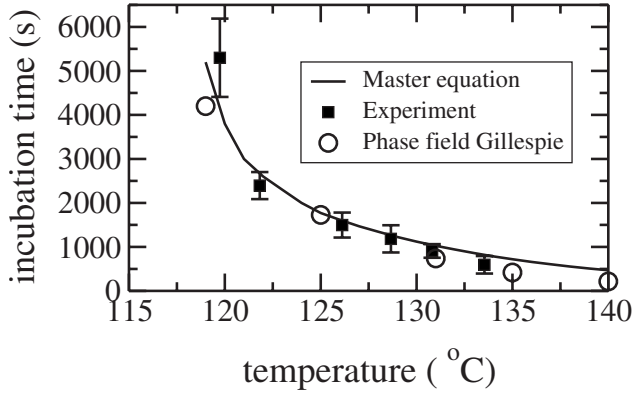

FIG. 4. Incubation times given by phase-field Gillespie simulations, with master equation simulations of GST crystallization from Ref. 5 and experimental data from Ref. 15 shown for comparison. Note that the phase-field Gillespie simulation produces a reasonable agreement with experiment.

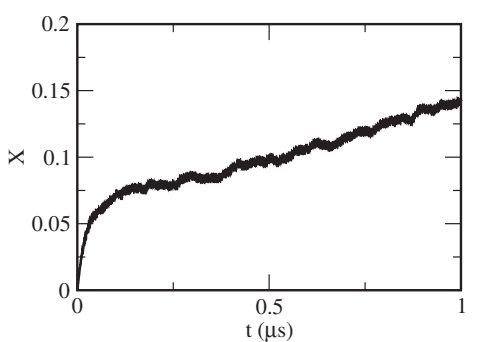

FIG. 5. Crystal fraction $X$ as a function of time starting at amorphous for $438 C$. Note that the appearance of small nuclei leads to the rapid growth in $X$ initially, but only the clusters past the critical size continue growing; others decay as there is a nontrivial nucleation size at this temperature.

data storage devices, as in Ref. 3. Such a material has a fine balance between bulk and surface energies of crystals, meaning that one can find nontrivial nucleation and growth dynamics that vary with $T$.

Let $T_{m}$ be the melting temperature; if we assume that the free energy change associated with crystallization of a single site varies linearly with $T-T_{m}$ and the energy change associated with a change in surface $A$ is $\sigma A$ with $\sigma$ as constant, then the rate $\xi(T, A)$ can be written as

$$
\xi(T, A)=\exp \left[L\left(1.0-\frac{T}{T_{m}}\right)-\frac{\sigma A}{k_{B} T_{m}}\right] .
$$

Following Ref. 13 we assume that

$$
L=\frac{\Delta H_{f} v_{m}}{2 k_{B} T_{m}},
$$

where constants are $\sigma=10^{-5} \mathrm{~J} \mathrm{~cm}^{-2}$ is the interfacial energy density between amorphous and crystalline phases and $S_{m}$ $=2.1187 \times 10^{-14} \mathrm{~cm}^{2}$ is the molecular surface area of the material. The values of $E_{a}=2.1 \mathrm{eV}$ and $k_{0}=10^{16} \mu \mathrm{s}^{-1}$ are also used in the current simulation. The other constants are as follows:

$\Delta H_{f}=625 \mathrm{~J} \mathrm{~cm}^{-3}$ is the enthalpy of fusion from the data obtained from differential scanning calorimeter experiments on GST.

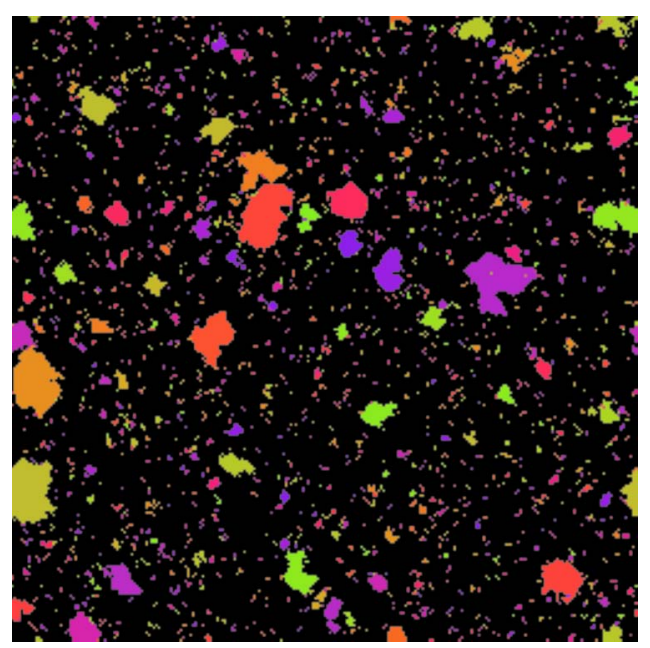

FIG. 6. (Color online) Crystal structure of a sample after subjecting to $1 \mu \mathrm{s}$ annealing at $438{ }^{\circ} \mathrm{C}$ from amorphous (i.e., the state at the end of the annealing shown in Fig. 5). Observe a small number of nucleated crystals that are growing, while other smaller crystals that are below nucleation size. 


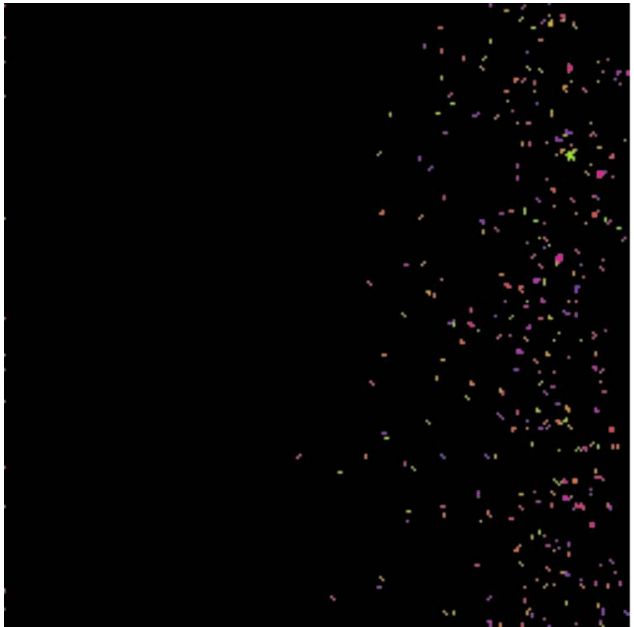

(a)

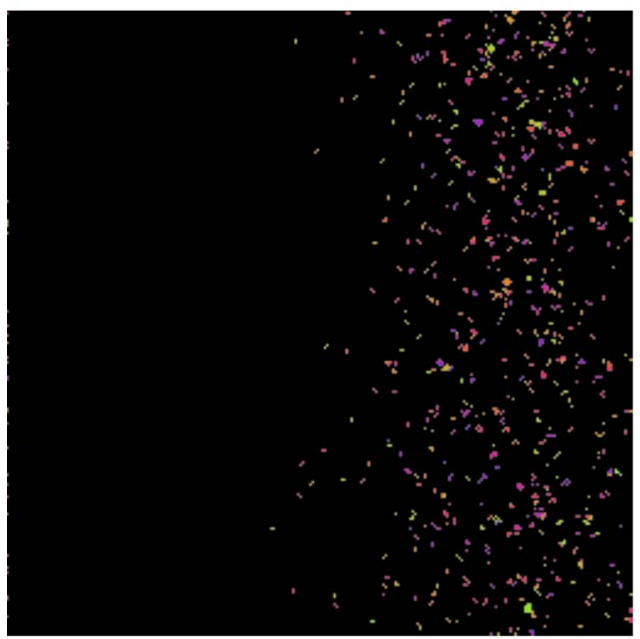

(b)

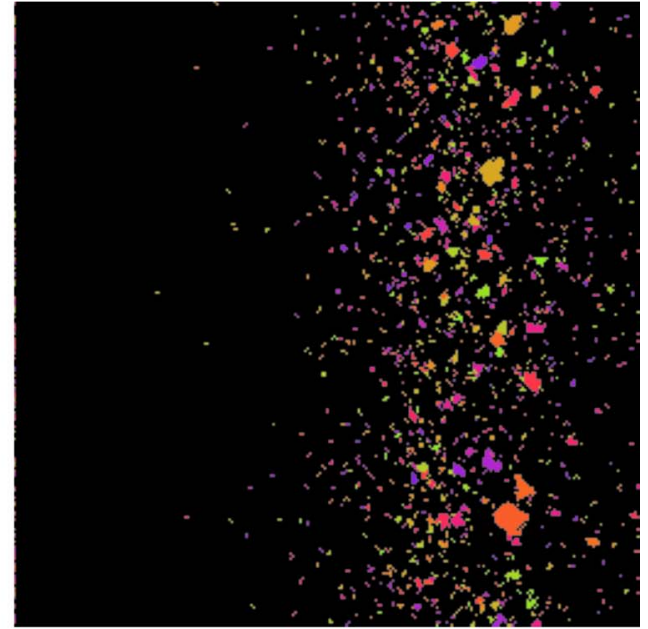

(c)

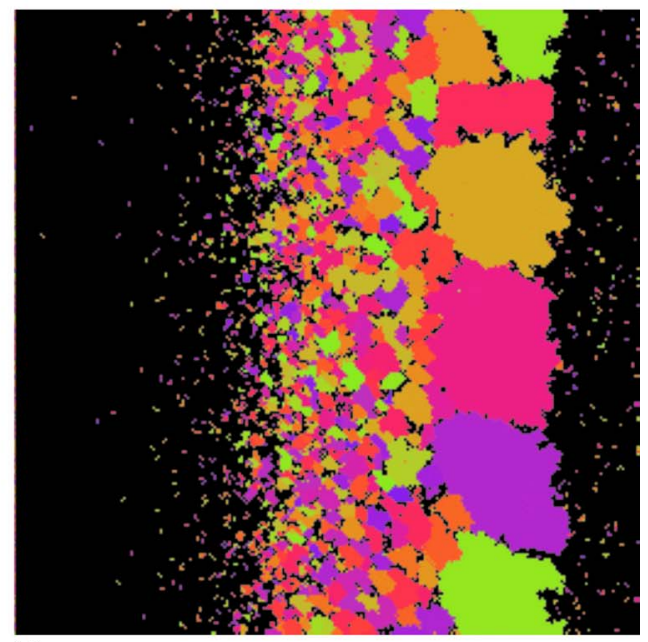

(d)

FIG. 7. (Color online) Images showing progress in crystallization for a sample held in a temperature gradient where the left boundary is $227{ }^{\circ} \mathrm{C}$ and the right is $477^{\circ} \mathrm{C}$. Observe the appearance of a band of higher crystallinity as time progresses from (a) after $17.6 \mathrm{~ns}$, (b) after $70 \mathrm{~ns}$, (c) after $554 \mathrm{~ns}$, and (d) after $22.9 \mu$ s. Observe that the effective nucleation size is larger on the right (hotter) side of the sample.

$v_{m}=2.9 \times 10^{-22} \mathrm{~cm}^{3}$ is the molecular volume of GST $T_{m}=889 \mathrm{~K}$ is the melting temperature,

$$
k_{B}=1.381 \times 10^{-23} \mathrm{~J} / \mathrm{K} .
$$

Using these values we obtain $L=7.381635$. In the present simulations, a grid of size $N \times N$ gives $N^{2}$ sites, on which periodic boundary conditions are applied in both directions; i.e. $r_{i+N j}=r_{i j+N}=r_{i j}$. The parameters for the GCA algorithm outlined above give realistic quantitative agreement with crystal growth in GST over a range of temperatures.

\section{A. Nucleation and crystal growth}

The simulations are performed by employing $N^{2}$ grid with $N=256$. Note that the crystalline fraction $X$ for such a grid can be calculated as $X=\left(1 / N^{2}\right) \sum_{i, j} r_{i j}$, where $0 \leq X \leq 1$ and $X=1$ corresponds to a fully crystalline state. The temporal increase in the crystalline fraction $X$ can be noticed in Fig. 1, starting at fully amorphous for $T=131{ }^{\circ} \mathrm{C}$; after an initial incubation the fraction quickly increases to saturate near fully crystalline. The insets show that the growth occurs subject to random fluctuations because of the Gillespie algorithm. Near $X=1$ there is still a nontrivial process of detachment and reattachment of sites from crystals that leads to grain coarsening over a long timescale. Figure 2 depicts the progress of this annealing at three stages; soon after inception, at approximately $20 \%$ progress and in a polycrystalline state, while Fig. 3 shows the development of the distribution of crystal sizes as the annealing progresses.

An incubation time (here taken to be the time to get to $20 \%$ crystallinity from fully amorphous) is shown against temperature in Fig. 4 along with for comparison the results from data from experiment ${ }^{15}$ as well as for the master equation model. ${ }^{5}$ It should be noticed that, the GCA simulations, while producing a less abrupt crystallization than the master equation model, are very close to the experimental results of Ref. 15 both in form and value. As in the master equation model, there is effectively only one fitting parameter in the model, the prefactor $k_{0}$ and this remains constant independent of temperature. The critical nucleation size results from a balance between volume and surface energies, when a crystallite grows in an amorphous matrix. This can be estimated 


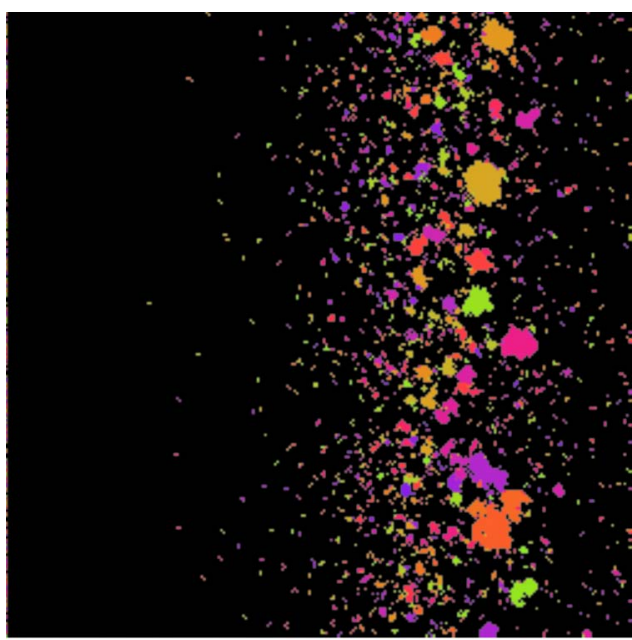

(a)

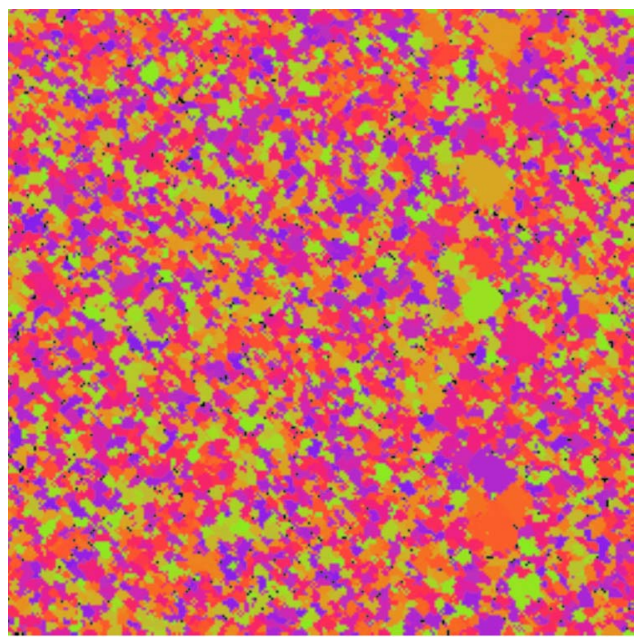

(b)

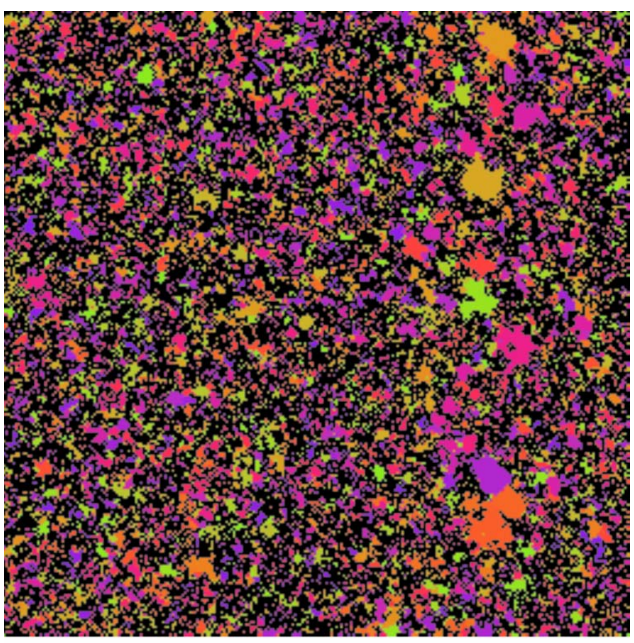

(c)

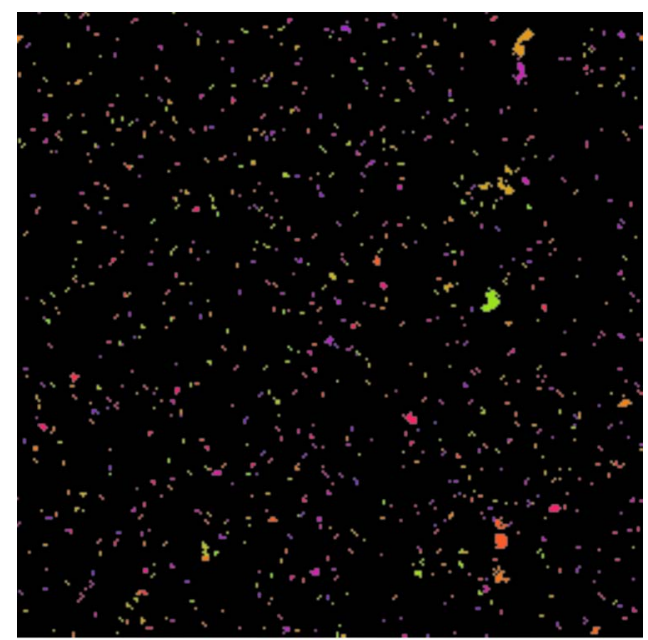

(d)

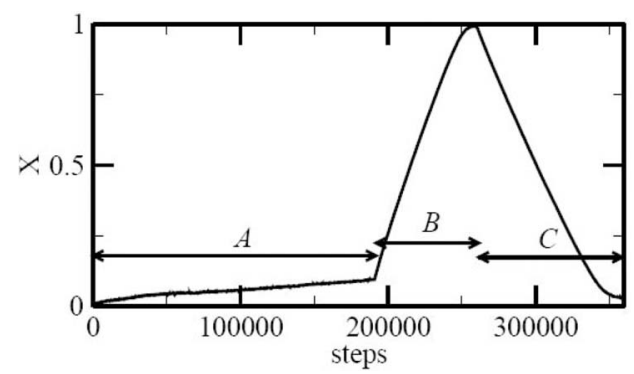

(e)

FIG. 8. (Color online) This figure shows the progress of a multistep annealing, demonstrating both spatial and temporal variations in temperatures. Starting from amorphous, the sample is first subjected to A, $1 \mu \mathrm{s}$ of a linear temperature gradient, the left at $227^{\circ} \mathrm{C}$ and the right at $477^{\circ} \mathrm{C}$; (a) shows the crystal structure. For the next $0.1 \mathrm{~s}$ it is subject to B, maintained at $227^{\circ} \mathrm{C}$, and in doing so progresses toward almost complete crystallization but with a clear banded structure; this is shown in (b). Finally the sample is subjected to $\mathrm{C}$ where it is raised to $477{ }^{\circ} \mathrm{C}$ for only $15 \mathrm{~ns}$ which is enough for the crystals to almost entirely dissociate; the structure at $X=0.5$ is shown in (c) and the final state is (d). Although below melting temperature, the critical nucleus size is too large for crystals of this size to survive. (e) shows the crystalline fraction $X$ as a function of algorithm step; note that the time intervals A-C vary over many orders of magnitude as the annealing progresses. 
using the JMAK formalism as outlined in Ref. 2, where the incubation time was estimated using Kashchiev. ${ }^{16}$ The equivalent in this model is obtained by noting that if we consider a disk-shaped crystallite incorporating $n$ sites, then the change in boundary area $A$ on adding one more site will scale proportional to $n^{-1 / 2}$. Hence the critical cluster size, where $\xi(T, A)=\xi^{-1}(T, A)$ will occur where $n=n_{\text {crit }}$ such that $n_{\text {crit }}=K\left(1-T / T_{m}\right)^{-1 / 2}$ for a constant $K$ that is in principle computable. In particular, one can observe from this calculation that $n_{\text {crit }} \rightarrow \infty$ as $T \rightarrow T_{m}$ in a qualitatively similar manner to that observed in Ref. 2.

\section{B. Nucleation and incomplete crystallization}

At higher temperatures the competition between growth and dissociation may lead to crystallization proceeding on a number of timescales, as illustrated in Fig. 5 and the corresponding crystal structure in Fig. 6 for the same material parameters as above but for a higher temperature. Note that the timescale is much faster; previous work has suggested that this mechanism may be responsible for the appearance of a "direct overwrite" regime in ovonic memory devices ${ }^{17}$ where a high temperature pulse can give rise to a resetting of the crystalline fraction to a given proportion. ${ }^{18}$

\section{Spatiotemporal annealings}

One can easily apply the algorithm to the case where the temperature, and therefore the rates of the reactions, depends on the spatial location; the algorithm is exactly as presented before except that $T$ now depends on site and time. As an example, in Fig. 7, the development of a band of GST material that is held at $227{ }^{\circ} \mathrm{C}$ on the left boundary and $477{ }^{\circ} \mathrm{C}$ on the right boundary is simulated. On the left hand side the growth is very slow while on the right the nucleation energy is difficult to overcome as it quickly leads to dissociation. Therefore, as expected, the growth is fastest in the intermediate region. A final example is given in Fig. 8 where a sample is subjected to a complex sequence of spatiotemporal annealings; see caption for details.

\section{DISCUSSION AND CONCLUSIONS}

A GCA model has been developed for the simulation of phase-transition processes in phase-change materials, such as GeSbTe alloys. The GCA approach incorporates the attractive features of a number of previous models for crystallization, and can be thought of as a thermodynamically motivated caricature of a molecular simulation. The GCA approach is potentially capable of spanning the length scales between $a b$ initio atomistic modeling methods and bulk-scale methods, and as such may be particularly suited to the realistic simulation and design of current and future phasechange based devices. Further investigations and enhancements of the model might focus on the following.

- Extension to a full 3D capability, potentially enabling the simulation of real device structures and important inhomogeneities (such as interfacial nucleation).

- The coupling of temperature evolution to predicted phase: this might be important since amorphous and crystal phases have different thermal, electrical, and optical properties that may introduce a phasedependent temperature distribution during electrical or optical heating.

- The energies of the crystallites do not depend on orientation in the current model. It would be relatively easy to include anisotropy, as it means crystallite growth rates would depend on orientation, as observed in many materials.

Even without such enhancements, the current model can evidently produce reasonably realistic and numerically efficient simulations of crystallization behavior during the complex spatiotemporal heating/cooling cycles used in phasechange memory applications. The GCA approach will thus be useful for modeling phase-change devices that use reversible transitions in GeSbTe and similar alloys to store and, perhaps in the future, process information. ${ }^{17,18}$ It might also provide a useful link between the atomistic modeling approaches and modeling on a length scale more suited to device engineering and design.

\section{ACKNOWLEDGMENTS}

We thank Konstantin Blyuss, Andrew Bassom, and Alexei Zaikin for discussions related to this project. We also thank the EPSRC-GB (via Grant Nos. GR/S31662/01 and EP/F015046/1) and the Leverhulme Trust (by way of Research Fellowship for CDW) for their support.

\footnotetext{
${ }^{1}$ A. Pirovano, A. L. Lacaita, A. Benvenuti, F. Pellizzer, and R. Bez, IEEE Trans. Electron Devices 51, 452 (2004).

${ }^{2}$ C. D. Wright, M. Armand, and M. M. Aziz, IEEE Trans. Nanotechnol. 5, 50 (2006).

${ }^{3}$ S. Senkader and C. D. Wright, J. Appl. Phys. 95, 504 (2004).

${ }^{4}$ E. R. Meinders, H. J. Borg, M. H. R. Lankhorst, J. Hellmig, and A. Mijiritskii, J. Appl. Phys. 91, 9794 (2002).

${ }^{5}$ K. B. Blyuss, P. Ashwin, A. P. Bassom, and C. D. Wright, Phys. Rev. E 72, 011607 (2005).

${ }^{6}$ Z. Sun, J. Zhou, and R. Ahuja, Phys. Rev. Lett. 96, 055507 (2006).

${ }^{7}$ J. Hegedus and S. R. Elliot, Nature Mater. 7, 399 (2008).

${ }^{8}$ W. Yu, C. D. Wright, S. P. Banks, and E. P. Palmiere, IEEE Proc. Sci. Measure. Tech. 150, 211 (2003).

${ }^{9}$ Y. Cao, D. T. Gillespie, and L. Petzold, J. Chem. Phys. 126, 224101 (2007).

${ }^{10}$ S. Vedantam and B. S. V. Patnaik, Phys. Rev. E 73, 016703 (2006).

${ }^{11}$ A. Karma and W.-J. Rappel, Phys. Rev. E 57, 4323 (1998).

${ }^{12}$ G. Caginalp and W. Xie, Phys. Rev. E 48, 1897 (1993).

${ }^{13}$ D. T. Gillespie, J. Phys. Chem. 81, 2340 (1977).

${ }^{14}$ S. S. Kim, S. M. Jeong, K. H. Lee, Y. K. Park, Y. T. Kim, J. T. Kong, and H. L. Lee, Jpn. J. Appl. Phys., Part 1 44, 5943 (2005).

${ }^{15}$ V. Weidenhof, I. Friedrich, S. Ziegler, and M. Wuttig, J. Appl. Phys. 89, 3168 (2001).

${ }^{16}$ D. Kashchiev, Surf. Sci. 22, 319 (1970).

${ }^{17}$ S. R. Ovshinsky, Jpn. J. Appl. Phys., Part 1 43, 4695 (2004).

${ }^{18}$ C. D. Wright, P. Ashwin, and K. Blyuss, Appl. Phys. Lett. 90, 063113 (2007).
} 